\title{
Correlative light microscopy for high-content screening
}

\section{Benjamin Flottmann ${ }^{1, *}$, Manuel Gunkel ${ }^{1, *}$, Tautvydas Lisauskas ${ }^{1, *}$, Mike Heilemann ${ }^{1,2}$, Vytaute Starkuviene ${ }^{1}$, Jürgen Reymann ${ }^{1}$, and Holger Erfle ${ }^{1}$}

\begin{abstract}
${ }^{1}$ BioQuant Centre, Heidelberg University, Heidelberg, Germany, and Insitute of Physical \& Theoretical Chemistry, Goethe University Frankfurt, Frankfurt, Germany
\end{abstract}

*B.F., M.G., and T.L. contributed equally to this work.

BioTechniques 55:243-252 (November 2013) doi 10.2144/000114099

Keywords: super-resolution microscopy; dSTORM; high-content screening; correlative microscopy; Golgi complex

High-throughput microscopy is an effective tool for rapidly collecting data on a large scale. However, high throughput comes at the cost of low spatial resolution. Here we introduce correlative light microscopy by combining fast automated widefield imaging, confocal microscopy and superresolution microscopy. We demonstrate the potential of this approach for scalable experiments. The workflow consists of a robust approach for selecting cells of interest on a wide-field screening microscope at low resolution and subsequently re-localizing those cells with micrometer precision for confocal and super-resolution imaging. As a case study, we visualized and quantified cis- and transGolgi markers at increasing resolution.
Method Summary: Quantitative description of biological systems is the major challenge of biological research. Fluorescence microscopy plays an important role because it allows researchers to not only visualize but also evaluate virtually every cellular process of interest. Fluorescence microscopy, however, comes with a trade-off between throughput and spatial/temporal resolution. Due to these technological limitations, imaging biological samples is generally constrained to either high throughput but low resolution or high resolution but low throughput.

\section{Introduction}

High-throughput widefield or spinning disk confocal screens can process large numbers of targets. For example, RNAi screens can be performed with a sample number sufficient to cover the whole human genome ( $\sim 23,000$ genes). To obtain a reasonable number of cells per field of view and to ensure fast imaging, low to medium spatial resolution is usually chosen $(10 \times$ air or $40 \times$ water objective) $(1,2)$.

Point scanning confocal microscopy is applicable to small-scale experiments and is often used following data collection by high-throughput methods (3). In order to increase the information content of small-scale confocal screens, automated correlative screening techniques (4), which combine low with high-resolution point scanning, may be used for targeting relevant phenotypes and zooming closer into interesting events by imaging in a mode of high information context.

The spatial resolution in both widefield and confocal microscopy is limited by diffraction. In recent years, a new generation of microscopy techniques has been developed that bypass this limit in resolution (5-7). Among these are single-molecule techniques, which were demonstrated to reach near molecular spatial resolution (8-12). Additionally, co-localization analysis can be refined by using super-resolution data. For example, co-localization analysis at the molecular level was demonstrated, attributing a co-localization value to each single biomolecule detected $(13,14)$.

In order to combine fast low-resolution widefield methods with slow high- or super-resolution microscopy, we have developed a workflow for correlative microscopy that combines the best features of each. By a fast widefield screen of a large sample, representative cells showing defined phenotypes are selected and subsequently imaged in confocal and super-resolution modes. To be able to image in the different microscopy modes, we have chosen fluorophores that can be used in all three imaging platforms. In this study, we use correlative light microscopy to analyze the distribution of proteins residing at different cisternae in the Golgi complex. We demonstrate significantly improved spatial resolution of GalT (trans-Golgi marker) and GM130 (cis-Golgi marker) under diverse treatments when applying correlative microscopy for the same cell.

\section{Materials and methods}

\section{Cell preparation}

NRK (normal rat kidney, CRL-6509; ATCC, Manassas, VA) cells that had been stably transfected with the Golgi enzyme GalT tagged with CFP (NRK-GalT-CFP) were cultured in DMEM (GIBCO/Invitrogen, CA) containing 5\% fetal bovine serum (FBS), $1 \%$ non-essential amino acids (GIBCO/Invitrogen), $2 \mathrm{mM}$ glutamine, 100 $\mathrm{U} / \mathrm{mL}$ penicillin and $100 \mu \mathrm{g} / \mathrm{mL}$ streptomycin. For experiments cells were plated on $\mu$-Slide 8-well slides (Ibidi, Martinsried, Germany) at densities of 15,000 cells/well $24 \mathrm{~h}$ before drug treatment. Poly-L-lysine(Sigma-Aldrich, M0) was applied to $\mu$-Slides for $15 \mathrm{~min}$ before cell plating. 


\section{Report}

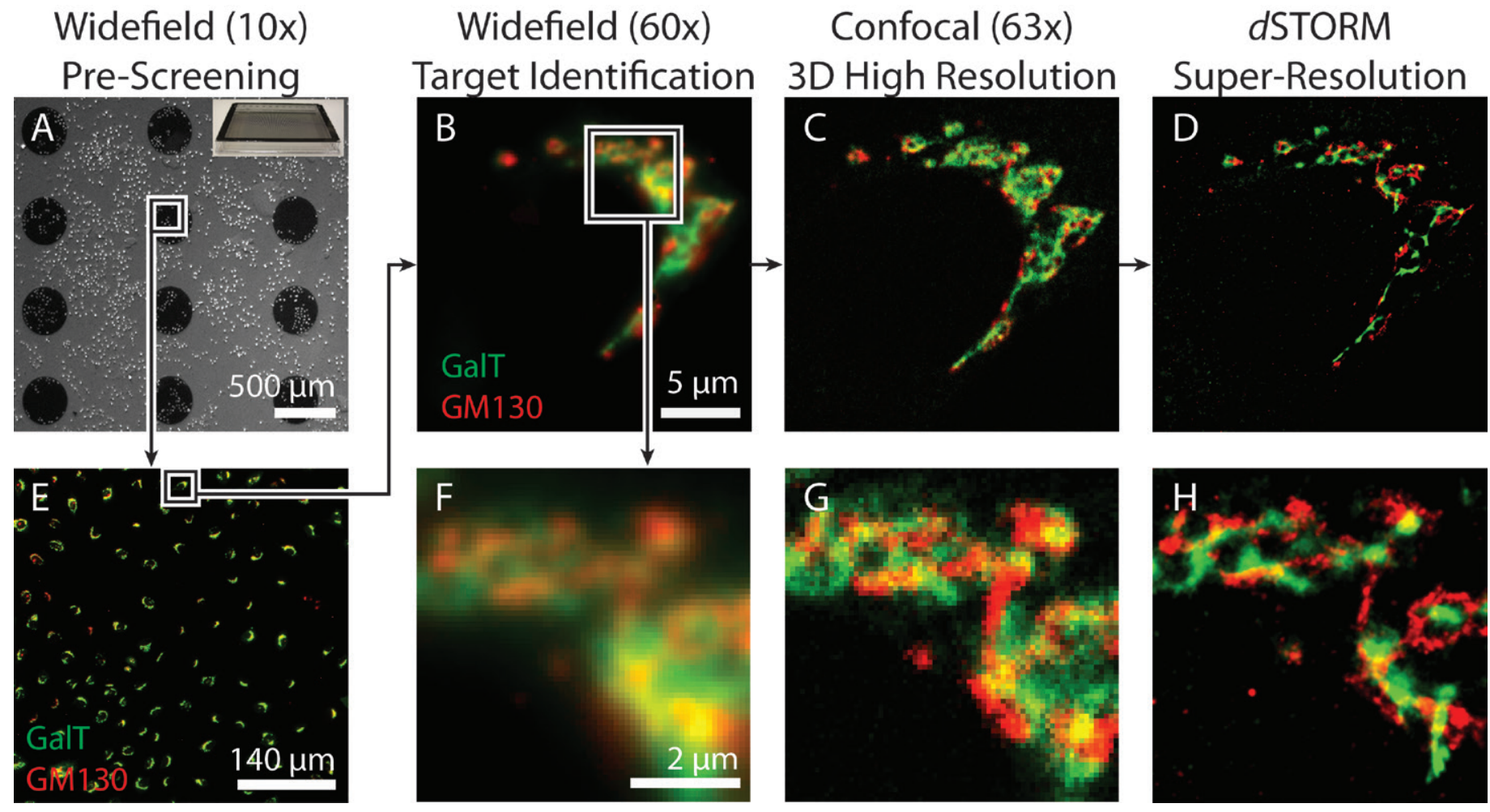

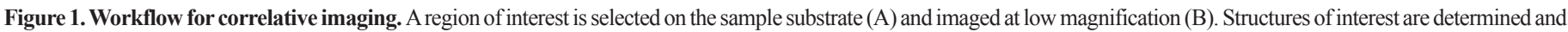

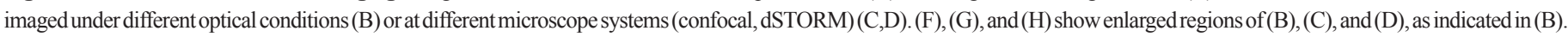

\section{Cell treatment}

BFA and nocodazole (Calbiochem/Merck Chemicals, Darmstadt, Germany) treatment was performed at concentrations of $5 \mu \mathrm{g} /$ $\mathrm{mL}$ and $1 \mu \mathrm{g} / \mathrm{mL}$, respectively, for $20 \mathrm{~min}$. Cycloheximide (0.1 mg/mL; Sigma-Aldrich) was added to abolish protein synthesis.

\section{Sample preparation and immunofluorescence}

Cells were fixed incubating in 3\% PFA for $20 \mathrm{~min}$. TetraSpeck microspheres $(0.1 \mu \mathrm{m}$; Invitrogen) were applied and incubated for $15 \mathrm{~min}$, and cells were permeabilized by incubation with $0.1 \%$ Triton-X-100 for 5 min. GalT-CFP was counter-stained with anti-GFP (6556, polyclonal rabbit; Abcam, Cambridge, UK) and anti-rabbit Alexa Fluor 532 (Invitrogen). GM130 was stained with anti-GM130 (monoclonal mouse; BD Biosciences, Franklin Lakes, NJ) and anti-mouse Alexa Fluor 647 (Invitrogen).

\section{Widefield microscopy}

Widefield images were acquired with an Olympus IX81 microscope with a 10× objective lens (Olympus UPlanSApo, NA 0.4; Olympus, Tokyo, Japan) and a field of view of $866 \mu \mathrm{m} \times 660 \mu \mathrm{m}$. The sample was illuminated by an $150 \mathrm{~W} \mathrm{Hg} /$ $\mathrm{Xe}$ mixed gas arc burner together with appropriate filter combinations for DAPI, Alexa Fluor 532, and Alexa Fluor 647, respectively. Integration times were set to $200 \mathrm{~ms}$ (20 ms for DAPI staining), which were the best match to the dynamic range of the CCD camera. In total, 50 fields of view were recorded for each cell selection.

\section{Confocal microscopy}

Selected cells were imaged on a Leica TCS SP5 with a $63 \times$ objective lens (Leica HCX PL APO, NA 1.40; Leica, Wetzlar, Germany), a scan speed of 400
$\mathrm{Hz}$ at $512 \times 512$ pixels and with a pixel size of $69 \mathrm{~nm}$, resulting in a field of view of $49 \mu \mathrm{m} \times 49 \mu \mathrm{m}$ (pinhole diameter of $96 \mu \mathrm{m})$. For each cell, 47 image planes with an axial distance of $0.2 \mu \mathrm{m}$ were acquired. Selected cells were initially positioned in the center of the z-stack by an autofocus routine before acquisition. Image acquisition took $196 \mathrm{~s}$ at each position, subsequent positions could be acquired every $212 \mathrm{~s}$ including positioning of the stage and autofocus routines. In total, confocal multicolor 3-D stacks were imaged at 796 single positions within $46.9 \mathrm{~h}$.

\section{Super-resolution imaging}

Super-resolution imaging of selected cells was performed with a custombuilt microscope using experimental protocols as described earlier, following direct stochastic optical reconstruction 
microscopy (dSTORM) (11). The beam of a multi-line argon-krypton laser (Innova70C, Coherent, Santa Clara, CA) is coupled into an acousto-optical tunable filter (27896; 8-channel) (AAOptics, Orsay, France) for wavelength selection and then combined with a UV laser beam (405 nm diode, Cube 405-100C, $100 \mathrm{~mW}$, Coherent). The combined beams are focused onto the back-focal plane of an oil-immersion total internal reflection fluorescence objective (PlanApo N 60×, NA 1.45, Olympus) mounted onto an inverted microscope (IX71; Olympus). Emission light is separated from excitation light by appropriate dichroic mirrors and filters (AHF, Tübingen, Germany) and focused on an electron multiplying charged coupled device (EMCCD) camera (Ixon, Andor, Ireland). The samples were mounted on the stage, and the sample chamber was filled with $\sim 200 \mu \mathrm{L}$ switching buffer (100 mM $\beta$-mercaptoethylamine; MEA in PBS, $\mathrm{pH} 8$ ). The exposure time was $50 \mathrm{~ms}$ with an electron multiplier gain of 200. Imaging intensities were $35 \mathrm{~mW}$ at $647 \mathrm{~nm}$ excitation wavelength and 65 $\mathrm{mW}$ at $532 \mathrm{~nm}$, respectively. To adjust the photoswitching rates of Alexa Fluor 647, illumination with UV light was gradually increased from zero intensity to a maximum intensity of $0.1 \mathrm{~mW}$. The two channels were recorded sequentially (first Alexa Fluor 647, followed by Alexa Fluor 532), chromatic shifts were corrected by using multi-color fluorescent beads (Tetraspeck, Invitrogen). $d$ STORM images with were typically reconstructed from 7000 frames using rapidSTORM (15).

\section{Relocation of structures of interest}

Relocation was done via coordinate transformation. In order to obtain a coordinate system on the sample that is independent of the coordinate system for the specific microscope system, we fixed three reference structures on the sample. These small pieces of titan coated glass, which each had a circular hole in the titan coating of $400 \mu \mathrm{m}$ in diameter, were imaged first in transmission mode. Their central positions ( $\mathrm{x}$ and $\mathrm{y}$ ) were determined by applying an automatic Otsu threshold and calculating the centroid of the resulting area. The $\mathrm{z}$ position was taken from the position of the $\mathrm{z}$-stage of the microscope after an autofocus routine. Based on these reference positions, an orthonormal basis of the sample was created, with the first reference position in the top left corner as origin. Widefield images of the sample were acquired, and the position of the lateral microscope stage and the axial z-drive was again recorded for each image. Individual cells of interest were selected for further imaging. The absolute position of these cells (position of the stage + position within the image) was transferred to the microscope stage independent coordinate system of the sample defined by the reference points. To transfer these coordinates to a second system, the referencing procedure was repeated and a coordinate transformation was performed.

\section{Cell selection}

Cellular positions for further confocal imaging were selected within the widefield images in two ways: 196 cells were selected manually by an expert marking the cellular positions within the image on a screen, and 600 cells were identified automatically based on nucleus segmentation within the images of the DAPI channel. The confocal images were then investigated for candidates for $d$ STORM acquisition and the appropriate cells were chosen manually.

Table 1. Experimental settings and parameters for different microscopy techniques.

\begin{tabular}{|lccc|} 
Microscope parameters & Widefield & Confocal & dSTORM \\
\hline Objective & $10 \times$ air, NA 0.4 & $63 \times$ oil, NA 1.4 & $60 \times$ oil, NA 1.4 \\
Field of view & $866 \mu \mathrm{m} \times 660 \mu \mathrm{m}$ & $49 \mu \mathrm{m} \times 49 \mu \mathrm{m}$ & $55 \mu \mathrm{m} \times 55 \mu \mathrm{m}$ \\
Pixel size & $645 \mathrm{~nm}$ & $69 \mathrm{~nm}$ & $107 \mathrm{~nm}$ \\
Imaging time (single plane) & $\sim 0.2 \mathrm{~s}$ & $\sim 1.28 \mathrm{~s}$ & $\sim 300 \mathrm{~s}$ \\
Duration of autofocus & $\sim 2 \mathrm{~s}$ & $\sim 5 \mathrm{~s}$ & $\mathrm{~N} / \mathrm{A}$ \\
\hline Estimated irradiation density & $<10 \mathrm{~W} / \mathrm{cm}^{2}$ & $<1 \mathrm{~kW} / \mathrm{cm}^{2}$ & One $-5 \mathrm{~kW} / \mathrm{cm}^{2}$ \\
\hline
\end{tabular}

\section{Correlative workflow}

Correlative image acquisition was performed as listed here:

1. Image reference markers on widefield setup

2. Image sample automatically on widefield setup

3. Identify target cells for confocal imaging manually / automatically

4. Image reference markers on confocal setup

5. Perform coordinate transformation of cell positions

6. Load position list and image cells automatically on confocal setup

7. Identify targets for $d$ STORM acquisition

8. Image reference markers on $d$ STORM setup

9. Perform coordinate transformation of cell positions

10. Load position list and image cells on dSTORM setup

\section{Results and discussion}

To achieve sequential correlative imaging on the same sample in three microscopy modes without additional sample preparation steps in between, we first tested various fluorophores for their suitability. Here, we demanded both high photostability and, in particular, the possibility of inducing photoswitching such that single-molecule super-resolution imaging, following the $d$ STORM protocol (11), was possible. We identified Alexa 


\section{Report}

Fluor 532 and Alexa Fluor 647 as an optimal pair for dual-color microscopy. Both fluorophores showed excellent photostability and were thus well suited for widefield and confocal imaging, as well as super-resolution imaging.

We set up a workflow as described in Materials and Methods to target structures of interest within low-resolution wide-field images, determine their spatial coordinates and relocate their positions on multiple other microscope systems. This workflow was applied here in the following way: After widefield imaging, we first transferred the sample to a confocal setup and then to a single-molecule localization microscope and imaged in these systems the same cells of interest identified in the widefield images. In widefield images at $10 \times$ magnification, cells of interest that showed the expected phenotype were selected and their positions (coordinates) were determined. The sample was then transferred to a confocal microscope. Using the coordinates, a confocal 3-D stack was acquired for each cell. The precision at which cells of interest could be re-localized was in the range of $10 \mu \mathrm{m}$, which was sufficient to position the whole cell of interest within the field of view of $\sim 50 \mu \mathrm{m}$. The sample was then passed on to the single-molecule localization microscope where selected cells of interest based on the confocal images were acquired. The re-localization of cells of interest here was slightly worse, since the reference structures could not be fitted within the field of view, and their center positions had to be estimated. However and more importantly, the cells of interest could easily be identified. Images acquired during this workflow are depicted in Figure 1 and illustrate the increase of resolution and information content achieved by this correlative approach. Imaging parameters for the different microscope techniques used are given in Table 1.

\section{Co-localization analysis in confocal and high-resolution imaging}

We then analyzed the capability of our correlative microscopy approach to compare the distribution of the cis- and trans-Golgi markers in NRK cells under diverse conditions $(16,17)$. Therefore, we imaged the cell samples using the workflow described above.

Our analysis proceeded as followed: Figure 2 shows the increased resolution
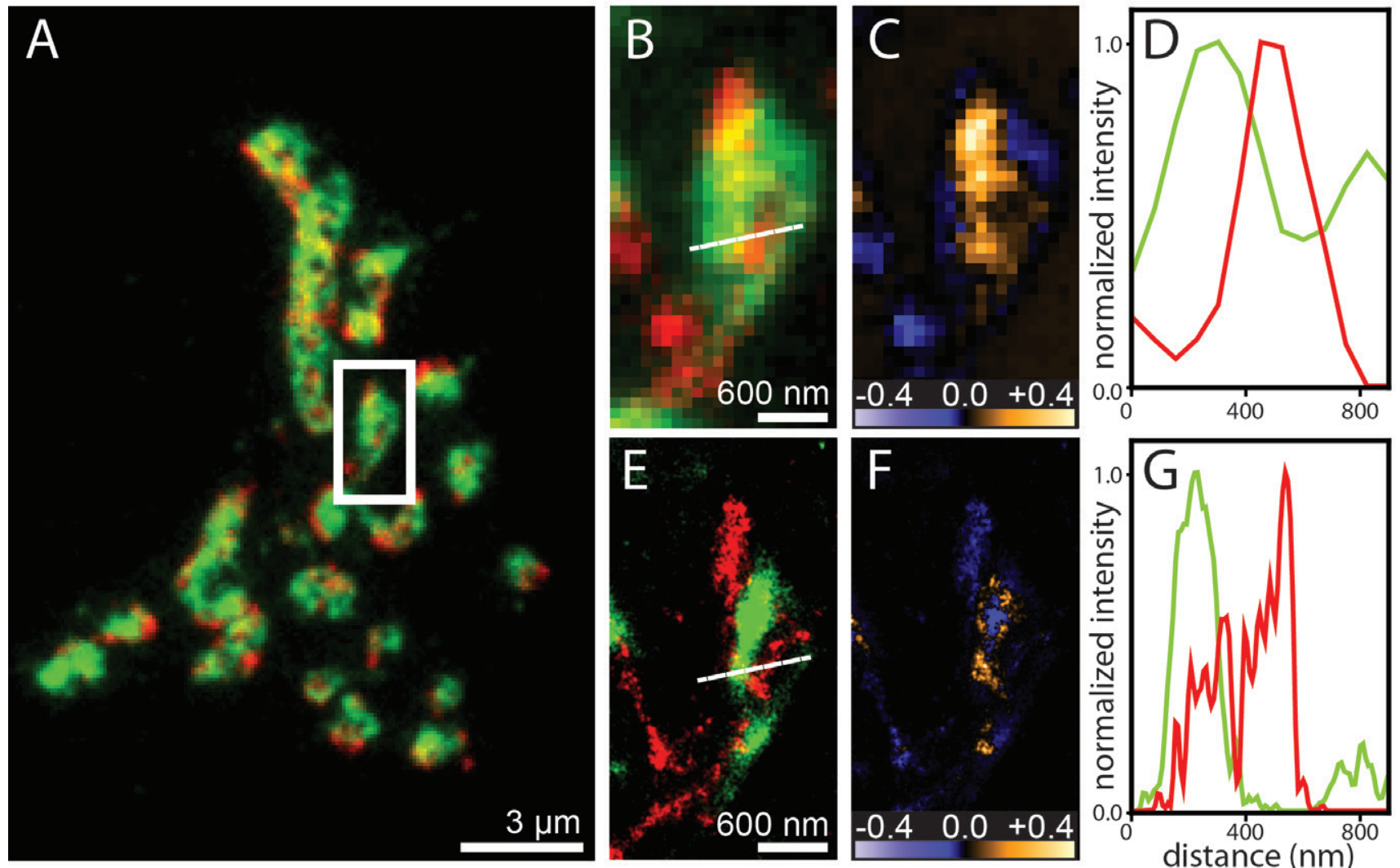

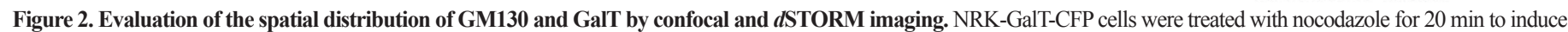

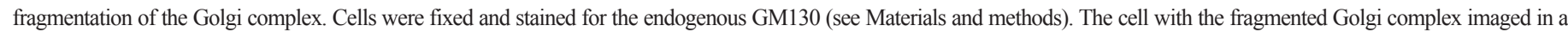

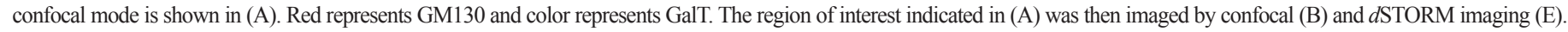

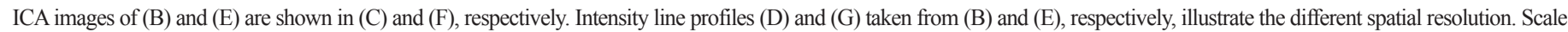

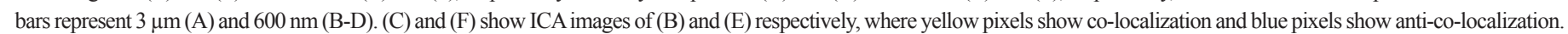




\section{Report}
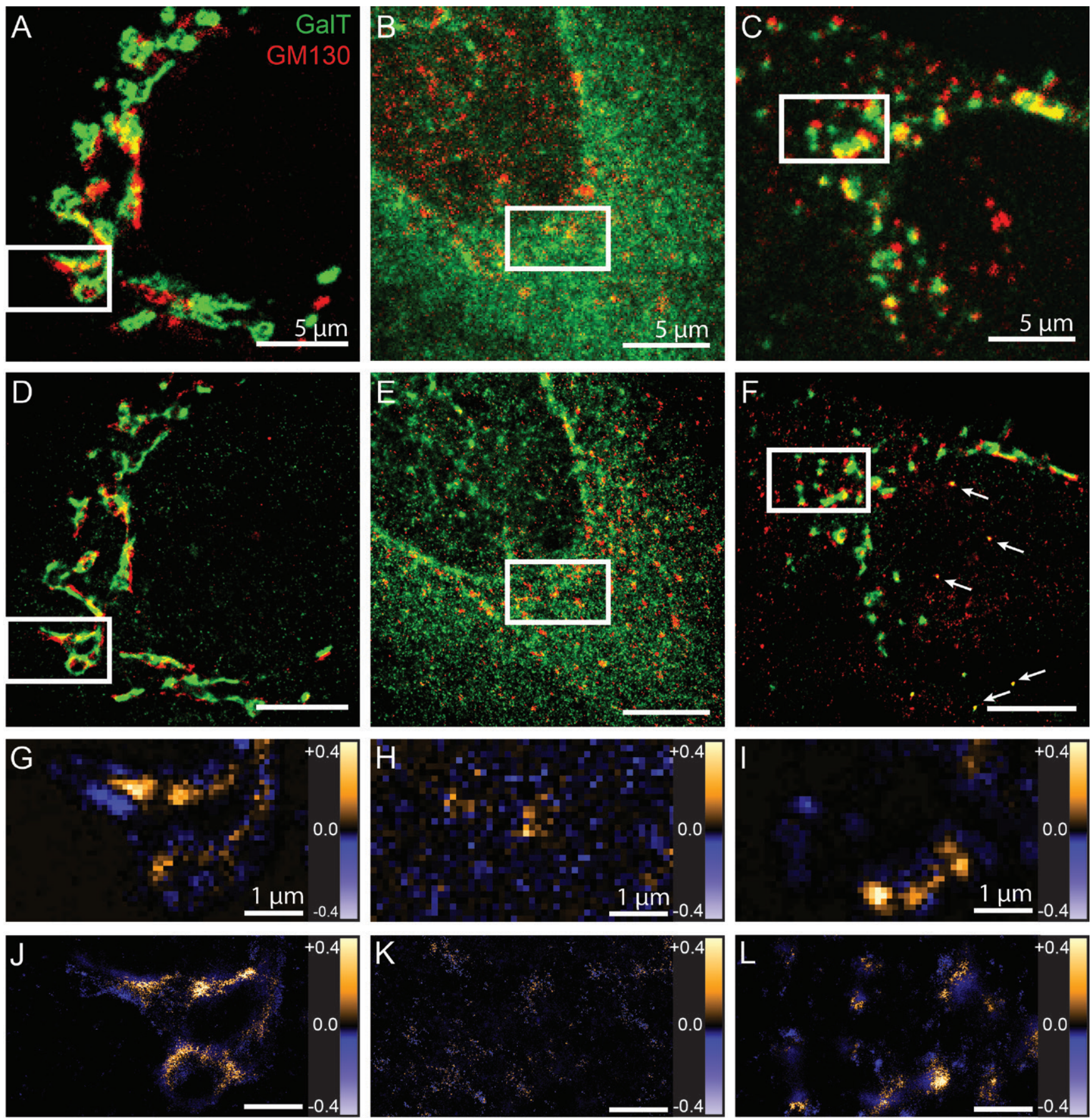

Figure 3. Distribution of GM130 and GalT under different treatments. GM130 and GalT were imaged in confocal (A, B, C) and dSTORM (D, E, F) mode. Red represents GM130 and green represents GalT. Intact Golgi complexes in the untreated cells are shown in (A) and (D), redistribution of the Golgi complexes after addition of BFA is shown in (B) and (E), fragmentation of the Golgi complexex after addition of nocodazole is shown in (C) and (F). The confocal images were analyzed for co-localization using ICA(G, H, I). The ICA analyses of the $d$ STORM images are shown in the bottom row $(\mathrm{J}, \mathrm{K}, \mathrm{L})$ (scale bars represent $5 \mu \mathrm{m}$ (fluorescence images) and $1 \mu \mathrm{m}$ (co-localization images)). Arrows in (F) indicate fiducial markers within the region of interest used for alignment of the red and green channels. More fiducial markers lie outside of selected regions and are not shown. 
complex to the endoplasmic reticulum (ER) (Figure 3B, E) $(19,20)$. In contrast to the experiments with nocodazole, where both GalT and GM130 had a similar localization pattern, GalT and GM130 showed a different behavior after BFA treatment. GalT was re-localized to the ER and formed a more reticular pattern, whereas GM130 appeared at the discrete ER exit sites, perceived as punctuate structures (21-24). Even if the separation of these two localization patterns is easily achieved by a confocal microscope (Figure 3B), $d$ STORM provides a more refined distribution, size and number of punctuate GM130 specific structures (Figure 3E). Similar to the experiments with nocodazole, a considerably improved co-localization of both markers is obtained (Table 2). The same is true for the untreated cells with an intact Golgi complex (Figure 3A, D). Spatial resolution of cisand trans-Golgi markers is increased nearly 2-fold according ICA analysis in $d$ STORM imaging compared with confocal images of the same cells (Table 2).

For the intact Golgi complex, co-localization analysis based on confocal images might be even more difficult due to the high densities of the markers (Figure 3A, G). Clearly, $d$ STORM in combination with ICA analysis is able to overcome these problems, particularly in the compact regions of the Golgi complex (Figure 3D, J). In addition, single-molecule based super-resolution techniques offer the additional advantage of providing molecular coordinates, which can be further processed in, for example, molecular-level co-localization analysis $(13,14)$. This is significantly different to co-localization analysis in confocal microscopy, which is based on the intensity information in individual pixels and thus is spatially constrained. In a coordinate-based approach applied to single-molecule localization data, a co-localization value can be attributed to each single molecule.

The correlative microscopy approach presented here combines fast widefield imaging of large sample numbers with high- and super-resolution microscopy, providing near-molecular level information on cellular structures. If certain structures of interest found in a widefield screen need further investigation by techniques with high resolution, the best representative regions can be chosen from the widefield images. The widefield imaging mode is then used as a filter or trigger system in order to identify predefined structures of interest and find the positions that are to be imaged in the other microscopy modes. This would be beneficial in high-throughput screening experiments, where a manual selection of cells for higher resolution imaging becomes quickly impractical due to the huge amount of data. Even in experiments where a sparse phenotype should be relocated and imaged in high-resolution systems, this approach can help by providing an image of the whole sample at low resolution. The high-resolution image can be recorded at exactly the right position by marking the appropriate spot in the low-resolution image. Due to the possibility of performing multiple experiments on the same cell, the information content is expanded, assuming that photobleaching is negligible (e.g., large structures with high concentrations of labeled proteins, short imaging times per region beforehand). Our workflow for relocating cells on different microscopic systems is furthermore not restricted to the fluorescence microscopic methods presented here, but is also applicable for the integration of any microscopic technique where the position of the reference markers can be identified.

\section{Author contributions}

The conception of the study, writing, and editing of the manuscript were done by all authors; the development of integrative microscopy approach and assay adaptation were done by B.F., M.G., J.R., T.L, V.S., M.H., and H.E. The study was executed by B.F., M.G., T.L., and J.R., ICA analysis of $d$ STORM images was performed by B.F.

\section{Acknowledgments}

We thank Sebastian Malkusch for help with co-localization analysis. This work was supported by contract research "Methoden für die Lebenswissenschaften" of the BadenWürttemberg Stiftung (grant nr. P-LS-SPII/11) and by the "New methods in systems biology" program of the Federal Ministry of Education and Research (grant nr. 0315523A). The
ViroQuant-CellNetworks RNAi screening facility is supported by the CellNetworksCluster of Excellence (EXC81).

\section{Competing interests}

The authors declare no competing interests.

\section{References}

1. Collinet, C., M. Stoter, C.R. Bradshaw, N. Samusik, J.C. Rink, D. Kenski, B. Habermann, F. Buchholz, et al. 2010. Systems survey of endocytosis by multiparametric image analysis. Nature 464:243-249.

2. Neumann, B., T. Walter, J.-K. Hériché, J. Bulkescher, H. Erfle, C. Conrad, P. Rogers, I. Poser, et al. 2010. Phenotypic profiling of the human genome by time-lapse microscopy reveals cell division genes. Nature 464:721727.

3. Osterwald, S., S. Wörz, J. Reymann, F. Sieckmann, K. Rohr, H. Erfle, and K. Rippe. 2011. A three-dimensional co-localization RNA interference screening platform to elucidate the alternative lengthening of telomeres pathway. Biotechnology Journal.

4. Conrad, C., A. Wünsche, T.H. Tan, J. Bulkescher, F. Sieckmann, F. Verissimo, A. Edelstein, T. Walter, et al. 2011. Micropilot: automation of fluorescence microscopy-based imaging for systems biology. Nat. Methods 8:246-249.

5. Hell, S.W. 2007. Far-field optical nanoscopy. Science 316:1153-1158.

6. Heilemann, M. 2010. Fluorescence microscopy beyond the diffraction limit. J. Biotechnol. 149:243-251.

7. Galbraith, C.G. and J.A. Galbraith. 2011. Super-resolution microscopy at a glance. J. Cell Sci. 124:1607-1611.

8. Betzig, E., G.H. Patterson, R. Sougrat, O.W. Lindwasser, S. Olenych, J.S. Bonifacino, M.W. Davidson, J. Lippincott-Schwartz, and H.F. Hess. 2006. Imaging intracellular fluorescent proteins at nanometer resolution. Science 313:16421645.

9. Rust, M.J., M. Bates, and X. Zhuang. 2006. Sub-diffraction-limit imaging by stochastic optical reconstruction microscopy (STORM). Nat. Methods 3:793-795.

10. Fölling, J., M.L. Bossi, H. Bock, R. Medda, C.A. Wurm, B. Hein, S. Jakobs, 
C. Eggeling, and S.W. Hell. 2008. Fluorescence nanoscopy by ground-state depletion and single-molecule return. Nat. Methods 5:943-945.

11. Heilemann, M., S. van de Linde, M. Schüttpelz, R. Kasper, B. Seefeldt, A. Mukherjee, P. Tinnefeld, and $M$. Sauer. 2008. Subdiffraction-resolution fluorescence imaging with conventional fluorescent probes. Angew. Chem. Int. Ed. Engl. 47:6172-6176.

12. Lemmer, P., M. Gunkel, D. Baddeley, R. Kaufmann, A. Urich, Y. Weiland, J. Reymann, P. Müller, et al. 2008. SPDM: light microscopy with single-molecule resolution at the nanoscale. Appl. Phys. B 93:1-12.

13. Malkusch, S., U. Endesfelder, J. Mondry, M. Gelléri, P.J. Verveer, and M. Heilemann. 2012. Coordinate-based co-localization analysis of single-molecule localization microscopy data. Histochem. Cell Biol. 137:1-10.

14. Gunkel, M., F. Erdel, K. Rippe, P. Lemmer, R. Kaufmann, C. Hörmann, R. Amberger, and C. Cremer. 2009. Dual color localization microscopy of cellular nanostructures. Biotechnol. J. 4:927-938.

15. Wolter, S., M. Schüttpelz, M. Tscherepanow, $S$. van de Linde, M. Heilemann, and M. Sauer. 2010. Real-time computation of subdiffraction-resolution fluorescence images. J. Microsc. 237:12-22.

16. Nakamura, N., C. Rabouille, R. Watson, T. Nilsson, N. Hui, P. Slusarewicz, T.E. Kreis,
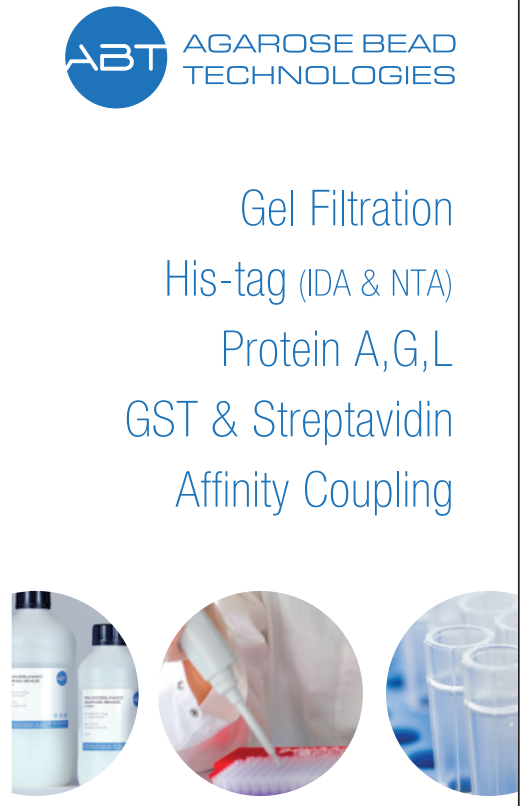

www.abtbeads.com

North America 8139082589 Europe / Asia + 34917610230 info@abtbeads.com and G. Warren. 1995. Characterization of a cis-Golgi matrix protein, GM130. J. Cell Biol. 131:1715-1726.

17. Röttger, S., J. White, H.H. Wandall, J.C. Olivo, A. Stark, E.P. Bennett, C. Whitehouse, E.G. Berger, et al. 1998. Localization of three human polypeptide GalNAc-transferases in HeLa cells suggests initiation of O-linked glycosylation throughout the Golgi apparatus. J. Cell Sci. 111:45-60.

18. Li, Q., A. Lau, T.J. Morris, L. Guo, C.B. Fordyce, and E.F. Stanley. 2004. A Syntaxin 1, Goo, and N-Type Calcium Channel Complex at a Presynaptic Nerve Terminal: Analysis by Quantitative Immunocolocalization. J. Neurosci. 24:4070-4081.

19. Klausner, R. and J. Donaldson. 1992. Brefeldin A: insights into the control of membrane traffic and organelle structure. The Journal of cell ...

20. Lippincott-Schwartz, J., L.C. Yuan, J.S. Bonifacino, and R.D. Klausner. 1989. Rapid redistribution of Golgi proteins into the ER in cells treated with brefeldin A: evidence for membrane cycling from Golgi to ER. Cell 56:801-813.

21. Tang, B., S. Low, and W. Hong. 1995. Differential response of resident proteins and cycling proteins of the Golgi to brefeldin A. - Abstract - UK PubMed Central. European journal of cell biology.

22. Mardones, G.A., C.M. Snyder, and K.E. Howell. 2006. Cis-Golgi matrix proteins move directly to endoplasmic reticulum exit sites by association with tubules. Mol. Biol. Cell 17:525-538.

23. Miles, S. 2001. Evidence that the entire Golgi apparatus cycles in interphase HeLa cells: sensitivity of Golgi matrix proteins to an ER exit block. J. Cell Biol. 155:543556.

24. Altan-Bonnet, N., R. Sougrat, W. Liu, E.L. Snapp, T. Ward, and J. Lippincott-Schwartz. 2006. Golgi inheritance in mammalian cells is mediated through endoplasmic reticulum export activities. Mol. Biol. Cell 17:990-1005.

Received 28 June 2013; accepted 10 October 2013.

Address correspondence to Holger Erfle, BioQuant Centre, Heidelberg University, Heidelberg, Germany, E-mail: holger. erfle@bioquant.uni-heidelberg.de, or Mike Heilemann, Goethe University Frankfurt, Frankfurt, Germany, E-mail: heilemann@ chemie.uni-frankfurt.de.

To purchase reprints of this article, contact: biotechniques@fosterprinting.com 